Hydrology and Earth System Sciences, 1(2), 257-264 (1997) C EGS

\title{
Towards a rational definition of potential evaporation
}

\author{
J.-P. Lhomme ${ }^{1}$ \\ CICTUS, Universidad de Sonora, AP 1819, Hermosillo, Sonora 83000, Mexico
}

\begin{abstract}
The concept of potential evaporation is defined on the basis of the following criteria: (i) it must establish an upper limit to the evaporation process in a given environment (the term 'environment' including meteorological and surface conditions), and (ii) this upper limit must be readily calculated from measured input data. It is shown that this upper limit is perfectly defined and is given by the Penman equation, applied with the corresponding meteorological data (incoming radiation and air characteristics measured at a reference height) and the appropriate surface characteristics (albedo, roughness length, soil heat flux). Since each surface has its own potential evaporation, a function of its own surface characteristics, it is useful to define a reference potential evaporation as a short green grass completely shading the ground.

Although the potential evaporation from a given surface is readily calculated from the Penman equation, its physical significance or interpretation is not so straightforward, because it represents only an idealized situation, not a real one. Potential evaporation is the evaporation from this surface, when saturated and extensive enough to obviate any effect of local advection, under the same meteorological conditions. Due to the feedback effects of evaporation on air characteristics, it does not represent the 'real' evaporation (i.e. the evaporation which could be physically observed in the real world) from such an extensive saturated surface in these given meteorological conditions (if this saturated surface were substituted for an unsaturated one previously existing). From a rigorous standpoint, this calculated potential evaporation is not physically observable. Nevertheless, an approximate representation can be given by the evaporation from a limited saturated area, the dimension of which depends on the height of measurement of the air characteristics used as input in the Penman equation. If they are taken at a height of $2 \mathrm{~m}$ (the height of the meteorological observations), the dimension of the saturated surface in the direction of the wind ranges roughly from 50 to $200 \mathrm{~m}$ for a short green grass completely shading the ground.
\end{abstract}

\section{Introduction}

It seems that the concept of 'potential rate' of evaporation appeared for the first time in a paper by Thornthwaite (1948) entitled 'An approach towards a rational classification of climate', where it was associated with the term 'evapotranspiration', which also appeared for the first time (Brutsaert, 1982; Monteith, 1985; Granger, 1989). Thornthwaite defines potential evapotranspiration as 'the amount of water which would transpire and evaporate if it were available', and he adds: 'When water supply increases, as in a desert irrigation project, evapotranspiration rises to a maximum that depends only on the climate. This may be called 'potential evapotranspiration', as distinct from actual evapotranspiration'. Since then, this concept has been used widely by hydrologists and agricultural meteorologists. However, a survey of the appropriate literature reveals that there have been several ways of defining the potential rate of evaporation or evapotranspiration, each one representing a different and distinct entity (Granger, 1989).

For instance, Penman (1956, p. 20) defines the concept of 'potential transpiration', (which, he writes, 'is unnecessarily expanded to 'potential evapotranspiration'), as 'the amount of water transpired in unit time by a short green crop, completely shading the ground, of uniform height and never short of water'. Some years later, to avoid considering oasis situations where strong advection from upwind surfaces can enhance potential transpiration independently of local meteorological factors, Penman (1963, p. 42) added the term 'extensive area' to characterize the size of the surface maintained at the potential rate, writing: '[potential transpiration or potential evapotranspiration] is expected to be a measure of the transpiration rate from an extensive short green cover completely shading the ground and adequately supplied with water'. About two decades later, Brutsaert (1982, p. 214) gives a similar definition: 'Potential evapotranspiration is now generally understood to refer to the maximum rate of evapotranspiration from a

\footnotetext{
${ }^{1}$ Permanent address: ORSTOM, Département Ressources et Environnement, 213 rue La Fayette, 75010, Paris, France.
} 
large area covered completely and uniformly by an actively growing vegetation with adequate moisture at all times', whereas Morton (1983, p. 15) states: 'The potential evapotranspiration, as estimated from a solution of the vapour transfer and energy-balance equations, represents the evapotranspiration that would occur from a hypothetical moist surface, with radiation absorption and vapour transfer characteristics similar to those of the area, and so small that the effects of the evapotranspiration on the overpassing air would be negligible'.

With regard to the concept of 'potential evaporation', WMO (1974) defines it as the 'quantity of water vapor which could be emitted by a surface of 'pure' water, per unit surface area and unit time, under the existing atmospheric conditions' (cited by Granger (1989)), whereas Perrier $(1977$, p. 9) defines the same concept as the evaporation from a stand when all its exchange surfaces are saturated: 'l'évaporation potentielle est naturellement mesurable après une pluie, une irrigation par aspersion ou en présence d'une forte rosée'. Brutsaert (1982, p. 214) gives approximately the same definition: 'Potential evaporation refers to the evaporation from any large uniform surface which is sufficiently moist or wet, so that the air in contact with it is fully saturated. Such conditions prevail usually only after the occurrence of precipitation and dew'. More recently, Shuttleworth (1993, p. 42) defines potential evaporation as 'the quantity of water evaporated per unit area, per unit time, from an idealized, extensive free water surface under existing atmospheric conditions', and Garratt (1994a, p. 125) states that 'it is defined as the maximum possible evaporation from a given surface for a given environmental state'.

Through this short review, it can be seen there exists a multitude of definitions concerning the concept of 'potentiality' applied either to evapotranspiration or evaporation. They differ substantially between themselves and can be quite contradictory, depending, for instance on the type of surface (free water, vegetation stand or any surface) or the size of the surface (extensive or small). However, usually (but not always), potential evaporation refers to the evaporation from free water, whereas potential evapotranspiration refers to the evaporation from a short green crop covering completely the ground and with adequate moisture. The confusion generated by all these definitions has been enhanced by the fact that leading evaporation scientists have criticized the term 'evapotranspiration' and recommended that it be abandoned in favour of the term 'evaporation'. For instance, Penman (1963, p. 33) writes: 'Many find it helpful to give a special name to the combined effect [some of the water coming directly from the soil and some coming indirectly from the soil through the plant], referring to it as 'evapotranspiration'. As it is used frequently, it is presumably a useful term, but it is rather ugly, and it hardly seems necessary, as there are few situations in which the use of 'evaporation' or 'transpiration' is not entirely adequate'. Monteith (1985, appendix) shares the same opinion: '. . . the word [evapotranspiration] is unnecessary. It is also inappropriate because its components are not strictly congruous; the word 'transpiration' implies a flux of vapour whereas the primary meaning of evaporation is a change of phase from liquid to gas'. Shuttleworth (1993, p. 41) seems to share also the same idea as he writes in the same sense: '. . . evaporation is defined as the rate of liquid water transformation to vapor from open water, bare soil, or vegetation with soil beneath', arguing that 'the physics of water vapor loss from open-water surfaces and from soils and crops is essentially identical'. In the following development, to limit the confusion, the term 'evaporation' will be employed systematically in the general sense proposed by Shuttleworth.

This brief 'historical' review shows that, in the field of hydrology and related sciences, the term 'potential' is not clearly understood when used to qualify evaporation, transpiration or evapotranspiration. The intent of this paper is to re-examine this concept of 'potentiality' in evaporation by a systematic approach and to try to clarify it. A rational definition of the potential rate of evaporation will be proposed on the basis of a sound criterion, and its physical and experimental significance will be examined, together with its relationship with actual evaporation.

\section{Defining and formulating potential evaporation}

It is essential to be precise about the purpose of potential evaporation before defining this concept. The point of view, shared by many authors over the last four decades (Garratt, 1994b), is that the main interest (and maybe only interest) is to establish an upper limit to the evaporation process in a given environment, the term environment including meteorological and surface conditions (evaporation depending on surface characteristics as well as on weather conditions). And for each set of meteorological and surface conditions, this upper limit must be unique, readily calculated from measured input data and, if possible, physically observable (i.e. measurable), because in the context of the practice of hydrology, it is useless to define a parameter which cannot be determined or measured easily. The determination of the upper limit to the evaporation process in a given environment needs a physical description of the process in question.

It is generally accepted that the description of the evaporation process $(E)$ of natural surfaces which is most physically sound is given by the Penman-Monteith onedimensional single source equation (Monteith, 1965), which reads as

$$
\lambda E=\frac{s\left(R_{n}-G\right)+\rho c_{p} D_{a} / r_{a}}{s+\gamma\left(1+r_{s} / r_{a}\right)}
$$

where $R_{n}$ is the net radiation, $G$ is the soil heat flux, $D_{a}$ is the vapour pressure deficit of the air at a reference height 
above the surface, $\lambda$ is the latent heat of vaporization, $\rho$ is air density, $c_{p}$ is the specific heat of air at constant pressure, $\gamma$ is the psychrometric constant and $s$ is the slope of the saturated vapour pressure curve at the temperature of the air $T_{a}$. The bulk aerodynamic is $r_{a}$ resistance between the surface and the reference height and $r_{s}$ is the bulk surface resistance to water vapour transfer. In conditions of neutral atmospheric stability, $r_{a}$ may be written as

$$
r_{a}=\frac{\ln ^{2}\left(z_{r} / z_{0}\right)}{k^{2} U_{a}}
$$

where $z_{0}$ is the roughness length, $U_{a}$ is the wind velocity at the reference height $z_{r}$, and $k$ is the von Karman constant $(k=0.4)$. The net radiation $R_{n}$ is detailed as

$$
R_{n}=(1-a) R_{s}+\varepsilon\left(R_{a}-\sigma T_{s}^{4}\right)
$$

where $R_{s}$ and $R_{a}$ are respectively the incoming solar and atmospheric radiations, $a$ is the surface albedo, $\varepsilon$ is the surface emissivity, $T_{s}$ is the surface temperature and $\sigma$ is the Stefan-Boltzmann constant. It is worthwhile pointing out that Eqn. (1) can be applied only in local advection-free conditions, when the surface fluxes are conservative on the path between the surface and the reference height (where climatic inputs are measured). It was derived initially for a homogeneous canopy completely covering the ground, but it can easily be extended to open water (by making $r_{s}=0$ ) or to bare soil (by reinterpreting the surface resistance). However, it is not entirely suitable for sparse crops partially covering the ground, for which a dual source model is generally needed (Shuttleworth and Wallace, 1985).

In this formulation (Eqn. (1)), the control of evaporation by the surface is exerted essentially through the surface resistance $r_{s}$. For given meteorological conditions (incoming radiations, vapour pressure deficit, air temperature and wind velocity), the lower the bulk surface resistance $\left(r_{s}\right)$, the higher the evaporation rate. The surface temperature will also change with variations in surface resistance, but it will affect evaporation in the same sense: the lower the surface resistance, the lower the surface temperature, which implies a greater net radiation and a lower soil heat flux and, consequently, a greater evaporation. As a first approximation in this analysis, the effect of surface temperature on the stability correction of $r_{a}$ will be neglected. So, taking into account the general significance to be given to the concept of potential evaporation, it is logical and sound to define it from Eqn. (1) by setting $r_{s}=0$, which corresponds to the highest rate of evaporation, all other conditions being equal. $r_{s}=0$ means that the vapour pressure of the air adjacent to the exchange surfaces (leaves, stems, soil, etc . . . ) is saturated, that is to say, the exchange surfaces are completely wet, as occurs after rainfall, dew deposit, or overhead irrigation. This definition corresponds to those proposed by Perrier (1977) and Brutsaert (1982) (mentioned in the introduction). Then, the potential rate of evaporation is expressed and determined by a Penman-type equation (Penman, 1948)

$$
\lambda E_{p}=\frac{s\left(R_{n}-G\right)+\rho c_{p} D_{a} / r_{a}}{s+\gamma}
$$

This equation shows that potential evaporation is a function of three types of parameter: (i) climatic inputs, which are incoming solar radiation $\left(R_{5}\right)$, incoming atmospheric radiation $\left(R_{a}\right)$, water vapour pressure deficit $\left(D_{a}\right)$, air temperature $\left(T_{a}\right.$, on which $s$ depends) and wind velocity ( $U_{a}$, on which $r_{a}$ depends); (ii) surface parameters, which are albedo $(a)$, emissivity $(\varepsilon)$, roughness length $\left(z_{0}\right.$, on which $r_{a}$ depends) and soil heat $\operatorname{flux}^{2}(G)$; (iii) an equilibrium term, the surface temperature, which adjusts itself to the climatic conditions as a function of surface and sub-surface (soil) characteristics. In a given meteorological environment, each type of surface has its own potential evaporation determined by its own surface characterisics.

According to the criteria presented above, the potential evaporation from a given surface in given meteorological conditions is defined by Eqn. (4), which will be further referred to as the Penman equation. ${ }^{3}$ It represents the evaporation rate from this surface (assumed to be extensive enough to obviate any effect of local advection), when completely wet and in the same meteorological environment. This physical representation is not in fact as evident as it seems at first glance. As pointed out by Shuttleworth (1993), this physical definition is purely conceptual and represents only an idealized situation, which cannot be encountered in the real world. This potential evaporation does not represent the 'real' evaporation (i.e. the evaporation which could be physically observed in the real world) from such an extensive saturated surface in the given meteorological conditions (assuming this saturated surface was substituted for an unsaturated one existing previously). This point will be thoroughly examined further.

At this stage, a first question arises. Since the above formulation shows there exist as many potential evaporations as surface types, what type of surface must be chosen as a reference to define the reference potential evaporation? It is relatively easy to answer this question. The evaporation from short green grass, completely shading the ground, and never short of water, has been universally used as a reference crop evaporation. Accordingly, it seems logical to

\footnotetext{
${ }^{2}$ Soil heat flux is considered as a surface characteristic although it is not strictly one. But this assimilation can be legitimized by the fact that for vegetation canopies covering the ground, it acts only as a corrective term to net radiation, and can be parameterized as a fraction of net radiation $G=$ $\alpha R_{n}$ (with for instance $\alpha \approx 0.05$ in the case of a short green grass).

${ }^{3}$ The Penman equation differs from the Penman formulae. The former is a physical equation, mathematically derived from basic equations describing the surface energy balance and the transfers into the atmosphere. It has a very general significance and can be applied to numerous situations. The latter are empirical formulae derived from the former. They are used in the practice of hydrology to calculate open water evaporation or potential evaporation from meteorological data.
} 
choose as a reference the potential rate of evaporation from such a crop. This choice appears to be more appropriate than the one of a 'free water surface', as stipulated by Shuttleworth (1993). The surface characteristics of open water ( $a$ and $z_{0}$, in particular) and the heat storage $(G)$ differ greatly from those of vegetation (to which most evaporation studies refer). Consequently, it is rather difficult to relate, theoretically, the actual evaporation from a crop or a natural vegetation to that from a free water surface. Moreover, in the case of free water, the problem of heat storage $(G)$ is crucial and rather difficult to resolve, as the temperature profile should be recorded as a function of depth. This experimental difficulty explains why the Penman equation is seldom used to estimate the loss of water from reservoirs, lakes or oceans (Monteith, 1981).

The criteria used by Granger (1989) to define potential evaporation are somewhat different from the one used in this development, because his definition has a different purpose: potential evaporation is seen as a simple index useful to estimate actual evaporation. He introduced as a basic criterion the fact that the relationship between potential and actual evaporation can be established, and he defines potential evaporation as 'the evaporation rate which would occur if the surface was brought to saturation and the atmospheric parameters and the surface temperature were held constant'. In the light of the formulation above, this definition appears to be unrealistic because it correponds to no physical or attainable situation: it is impossible to imagine a given surface which, under the same atmospheric conditions, would remain at the same temperature, when passing from an unsaturated state to a saturated one. Moreover, to maximize the evaporation rate in saturated conditions, it is not necessary to keep the surface temperature constant: the diminution of temperature resulting from a wetting of the surface leads to increasing the available energy $\left(R_{n}-G\right)$ with respect to the non-saturated case, and therefore to increasing the evaporation rate. Another argument against this definition is that surface temperature is rarely available.

\section{The physical significance of potential evaporation}

From a theoretical point of view, potential evaporation is defined on the basis of Penman's formulation and establishes an upper limit to the evaporation process in a given environment (the surface being saturated so that $r_{s}=0$ ). The question which arises now is to whether this theoretical limit has any meaning in the real world, i.e., if it is physically observable, and if it is, what should be the size of the saturated surface? In the different definitions of potential evaporation (or evapotranspiration) encountered in the literature, some specified that the surface should be extensive, whereas Morton (1983) specified the contrary. In an idealized world, the surface must be extensive enough to prevent any local advection related to leadingedge effects; in the real world, should the saturated surface be extensive?

This point is linked closely to the fact that the characteristics of the air near the surface are not external factors completely independent of surface conditions (and the evaporation process in particular) but, partially a consequence of the surface energy balance. If the problem is limited to one of large scale (or minimal) advection (excluding situations of local advection), there exists a feedback relationship between meteorological variables and evaporation process which cannot be ignored if potential evaporation has to correspond to a definable physical situation, observable and measurable. Due to this feedback effect, the area of the surface maintained at potential rate should not be too extensive. If it were, the measured air characteristics, which serve as inputs to the Penman equation, would be altered by the existence of this saturated surface, and would not be the same as those required by the definition. In this respect, Brutsaert (1982) writes: '. . . potential evaporation is often calculated by means of meteorological data observed under non-potential conditions. Clearly, this is not the same rate as that which could be calculated (or observed) if the surface had been adequately supplied with water ... The partition of the available energy at the surface . . . affects the temperature, the humidity and other state variables of the atmosphere.' And Nash (1989) gives the following illustration: 'Irrigation of the Sahara desert would produce complex changes in the atmosphere which would themselves affect the potential evaporation .... . To neglect the feedback effect would lead to the obviously incorrect conclusion that the actual evaporation from an irrigated Sahara would equal the present potential rate'. In the appendix, this feedback effect at regional scale is explored theoretically in a simple way using a closed-box model of the Convective Boundary Layer (CBL). It is important to stress that this problem of size arises only because potential evaporation is generally estimated from meteorological inputs measured in nonpotential conditions. If potential evaporation is estimated from weather data measured over a saturated area, the problem no longer exists. Consequently, if the area at potential rate cannot be too extensive, what must be its size? Another way to formulate the problem is to wonder if potential evaporation, calculated with meteorological data measured in non-potential conditions, is physically observable.

As a first approximation, the incoming radiations ( $R_{s}$ and $R_{a}$ ) do not depend on the height at which they are measured, and they can represent local values as well as regional values. However, air characteristics such as water vapour pressure deficit $\left(D_{a}\right)$, air temperature $\left(T_{a}\right)$ and wind velocity $\left(U_{a}\right)$ are largely dependent on the height of measurement, at least within the first meters above the ground. Clearly, the potential evaporation calculated with these input data will correspond to a different physical situation 
depending on the measurement height. Meteorological observations are generally made at a height of $2 \mathrm{~m}$ above a stand of short grass, this height being representative of local conditions. From an experimental viewpoint, this calculated potential evaporation will represent physically the evaporation from a saturated surface small enough for the excess moisture flux (and the possible different radiative and aerodynamic properties, if the saturated surface is different from the one previously existing) not to alter the air characteristics measured at the screen height $(2 \mathrm{~m})$ and in equilibrium with the actual evaporation. At the same time, this saturated surface must be large enough so that the depth $\left(h_{b}\right)$ of its Internal Boundary Layer (IBL) can reach the measurement height $(2 \mathrm{~m})$ so that the fluxes of sensible and latent heat can be assumed to be conservative between the surface and the reference height (which is a basic condition for deriving the Penman-Monteith equation). It is typically a problem of local advection (related to a discontinuity of the surface) and of internal boundary layer growth (Brutsaert, 1982).

Within the IBL exists an inner or equilibrium layer (of depth $h_{s} \approx 0.1 h_{b}$ ), where the profile characteristics are completely adjusted and fully governed by the local boundary conditions (with a logarithmic form in neutral conditions), and where fluxes are conservative (Garratt, 1994a). Above this equilibrium layer, and within the IBL, there is a blending layer in which the velocity distribution changes gradually from downstream characteristics to upstream characteristics. Strictly speaking, it is impossible to find, in the IBL, a region where air characteristics are the same as those upwind and where the profiles are conservative. For the first criterion to be met, the region of measurement should be above the IBL, and for the second criterion, the region of measurement should be just at the top of the equilibrium layer. Consequently, from a strictly physical standpoint, potential evaporation calculated in nonpotential conditions is not experimentally observable.

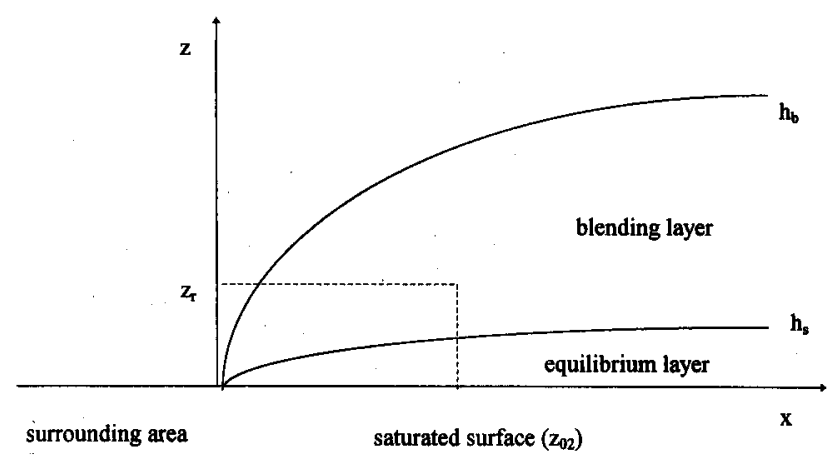

Fig. 1 Schematic diagram illustrating the development of the Internal Boundary Layer (IBL) resulting from a surface discontinuity. $h_{b}$ is the height of the $I B L ; h_{s}$ is the height of the equilibrium or inner layer; $z_{r}$ is the screen height where meteorological measurements are made.
Nevertheless, a compromise can be proposed by admitting that air characteristics measured within the blending layer represent an approximation which meets both criteria (Fig.1). To obtain an order of magnitude of the downward dimension of the saturated surface, one can use for instance the table given by Garratt (1994a, p.113), which shows the fetch $x$ required to obtain an Internal Boundary Layer of specified height in the case of a roughness change. The values are calculated from an equation derived by Miyake (1965)

$$
\left(h_{b} / z_{02}\right)\left[\ln \left(h_{b} / z_{02}\right)-1\right]+1=A x / z_{02} \text { with } A=0.5
$$

According to this equation, the fetch $x$ needed to reach an IBL of specified depth $h_{b}=2 \mathrm{~m}$ for a downwind stand of grass (of roughness length $z_{02}=0.01 \mathrm{~m}$ ) is equal to $17 \mathrm{~m}$. If, instead of the depth of the IBL, the depth $h_{s}$ of the equilibrium layer is considered, a fetch of $264 \mathrm{~m}$ is needed to reach $2 \mathrm{~m}$. Consequently, if potential evaporation is calculated from meteorological measurements at $2 \mathrm{~m}$, the stand of grass maintained at potential rate must have actually a downwind dimension of this order of magnitude, say, between 50 and $200 \mathrm{~m}$. Thus, the meteorological conditions will not be affected unduly by the surface conditions at saturation and the convective fluxes will be approximately conservative between the surface and $2 \mathrm{~m}$. From an experimental standpoint, the potential evaporation calculated in this way represents approximately the evaporation that could be measured at this distance ( 50 to $200 \mathrm{~m}$ ) from the leading edge. It is a rough approximation, but only a compromise is achievable.

\section{Potential evaporation and actual evaporation}

The theoretical relationship between the actual evaporation from a given vegetation and the reference potential evaporation (from a short green grass) can be inferred from Eqns (1) and (4). Using the prime symbol to denote the variables related to the reference potential evaporation, and putting $\eta=\left(R_{n}-G\right) /\left(R_{n}-G\right)^{\prime}$, the ratio of the available energy of the given vegetation to that of the reference vegetation at potential rate, and $\nu=r_{a} / r_{a}$, the ratio between the aerodynamic resistances of these two vegetations, the actual evaporation from the given vegetation can be expressed as

$$
\lambda E=\frac{\eta\left[s\left(R_{n}-G\right)^{\prime}\right]+v\left[\rho c_{p} D_{a} / r_{a^{\prime}}\right]}{s+\gamma\left(1+r_{s} / r_{a}\right)}
$$

When the vegetation considered has the same (or approximately the same) available energy and the same (or approximately the same) aerodynamic resistance as the reference vegetation at potential rate (which means that $\eta=\nu=1$ ), Eqn. (6) turns into 


$$
E=k . E_{p} \text { with } k=1 /\left(1+\frac{\gamma}{s+\gamma} \frac{r_{s}}{r_{a}}\right)
$$

$E_{p}$ being the potential evaporation as defined by Eqn. (4). Equation (7) shows that actual evaporation is proportional to potential evaporation. In this sense, potential evaporation appears to be a cause or a forcing variable, and therefore a positive index of actual evaporation. The coefficient $k$ is a mixture of surface properties and climate characteristics: it depends not only on the bulk surface resistance $r_{s}$ (which is a function of LAI and leaf stomatal resistance), but also on wind velocity (through $r_{a}$ ), surface roughness (through $r_{a}$ also) and air temperature (through $s$ ). It is worthwhile stressing that Eqn. (7) can never be completely true, even when it refers to the same vegetation (albedo and roughness length being identical): when passing from a saturated state to a non-saturated state, the surface temperature increases; this modifies both the net radiation (through the out-going long-wave radiation) and the aerodynamic resistance (through the correction for instability).

Most crop water requirements studies (Doorenbos and Pruitt, 1977) are based on the concept of reference crop evaporation $\left(E_{r c}\right)$, which corresponds to the definition of 'potential evapotranspiration' as given by Penman (1956), and which Shuttleworth (1993) defines in a more physical way as 'the rate of evaporation from an idealized grass crop with a fixed crop height of $0.12 \mathrm{~m}$, an albedo of 0.23 , and a surface resistance of $69 \mathrm{~s} \mathrm{~m}^{-1}$. The aerodynamic resistance $r_{a}$ of the reference crop is given by $208 / U_{a}$ in SI units, $U_{a}$ being the wind speed measured at $2 \mathrm{~m}$ (Shuttleworth, 1993, p.4.13). Consequently, the potential evaporation from the reference crop, denoted by $E_{p}$ (reference), is expressed in SI units as

$$
\lambda E_{p}(\text { reference })=\left[s\left(R_{n}-G\right)+\left(\rho c_{p} U_{a} D_{a} / 208\right)\right] /(s+\gamma)
$$

with $\gamma=67 \mathrm{~Pa}^{\circ} \mathrm{C}^{-1}$ and $c_{p}=1013 \mathrm{~J} \mathrm{Kg}^{-1}{ }^{\circ} \mathrm{C}^{-1} . \lambda$, s and $\rho$ vary with temperature (cf. equations given by Shuttleworth (1993)). The relationship between the reference crop evaporation $E_{r c}$ and $E_{p}$ (reference) is readily obtained from Eqn. (7), since the assumption of a same available energy and a same aerodynamic resistance can be justified as a first approximation. Replacing $r_{s}$ by 69 , one obtains

$$
\begin{aligned}
& E_{r c}=k_{r c} . E_{p}(\text { reference }) \text { with } \\
& k_{r c}=1 /\left[1+\frac{\gamma}{s+\gamma}\left(0.33 U_{a}\right)\right]
\end{aligned}
$$

where $k_{r c}$ depends only on climate inputs (wind speed and air temperature through $s$ ). This simple relationship (Eqn. (9)) between reference crop evaporation and reference potential evaporation could not have been derived, were potential evaporation defined as the evaporation from a free water surface. In this respect, Thom and Oliver (1977) examined the ways of modifying the original Penman formula for open water (Penman, 1948), in order to obtain reliable estimates of 'potential evapotranspiration' for short vegetation. They showed that the wind function used in the standard Penman formula (empirically derived from measurements with a particular open water surface) underestimated conductance for arable crops and forests. In fact, the new equation they recommend is equivalent and very close in form to Eqn. (9), both being obtained from the Penman-Monteith formulation (Eqn. (1)). The crop factor, commonly used in the practice of agronomy and irrigation, relates the maximum actual evaporation $\left(E_{m}\right)$ (i.e. with optimum soil water) from a given crop $(c)$ at a given phenological stage, to the reference crop evaporation: $k_{c}=E_{m}(c) / E_{r c}$ (Doorenbos and Pruitt, 1977). Wallace (1995) examined the theoretical expression of crop factors $k_{c}$. He showed that they are not the universal physiological constants that they are sometimes assumed to be, and that better progress would be made by using surface resistance.

Priestley and Taylor (1972) analysed an exhaustive set of evaporation data for open water and well-watered vegetation, measured in advection-free conditions, in terms of the quantity $\alpha=E / E_{0}$, where $\lambda E_{0}=s\left(R_{n}-G\right) /(s+\gamma)$ is the equilibrium evaporation obtained when a fully wet surface evaporates into a saturated atmosphere. Making no clear difference between $E_{p}$ and $E_{r c}$, they showed that in most conditions the second term in Eqn. (8) was about 20 to $30 \%$ larger than the first term. They concluded that 'potential evaporation' could be expressed as $\alpha E_{0}$, where $\alpha$ had a recommended value of 1.26 . Since then, it has been confirmed that this value of $\alpha$ is applicable in humid climates, but that a much higher value must be used in arid climates (Jensen et al., 1990). The additional energy, implied by a factor $\alpha$ greater than 1, has been attributed to the entrainment of relatively warm, dry air downwards through the top of the convective boundary layer. Recently, Lhomme (1997) gave an alternative explanation involving the feedback of regional evaporation on local potential evaporation (cf. appendix).

\section{Conclusion}

The definition proposed for potential evaporation can be worded in the following way. The potential evaporation from a given surface (vegetation, bare soil or open water), under given meteorological conditions, is defined as the upper limit of evaporation from this surface in these meteorological conditions. This upper limit exists, and occurs when the surface is completely wet (as after rainfall, dew deposit or irrigation by sprinkling). It can be estimated from the Penman equation Eqn. (4) with the appropriate surface albedo, soil heat flux and roughness length. A short green cover, completely shading the ground and with all its foliage wet, can be recommended as a reference surface to define the reference potential evaporation. 
In an idealized world, potential evaporation represents the evaporation from a saturated surface, extensive enough to cancel any effect of local advection, with the same characteristics as the given surface and under the same meteorological conditions. By no means does it represent the 'real' evaporation (i.e. the evaporation which could be physically observed in the real world) from such an extensive saturated surface in these given meteorological conditions (if this saturated surface were substituted for an unsaturated one previously existing). From a strictly physical standpoint, potential evaporation calculated in nonpotential conditions from Penman's equation is not experimentally observable. However, an approximate representation can be given by a saturated surface of limited area the dimension of which will depend on the height at which the air characteristics used as input are measured. If they are taken at a height of $2 \mathrm{~m}$ (screen height) and, in the case of a grass stand, the dimension of the saturated surface in the direction of the wind ranges roughly between 50 and $200 \mathrm{~m}$, the calculated evaporation represents approximately the evaporation which could be measured at this distance from the leading edge.

To prevent any confusion between potential evaporation and potential evapotranspiration following Shuttleworth (1993) and Wallace (1995), the widely used concept of 'potential evapotranspiration', defined according to Penman $(1956,1963)$ (as the transpiration rate from a short green cover completely shading the ground and adequately supplied with water in a given meteorological environment), could be labelled as 'reference crop evaporation' or simply 'reference evaporation' and noted $\left(E_{r c}\right)$. It can be estimated from Eqn. (9). The physical and experimental significance of this evaporation raises the same problem as that encountered for potential evaporation. In an idealized world, it represents the evaporation from an area extensive enough to obviate any effect of local advection. But in the real world, it represents the evaporation from a limited area, to avoid the feedback effect of surface energy balance on meteorological variables.

\section{Appendix: The feedback at regional scale and the Priestley-Taylor coefficient}

The Convective Boundary Layer (CBL) is the turbulent layer of the atmosphere which develops from the ground upwards because of the convective motions generated by the sensible heat flux released at the surface during the daytime (de Bruin, 1989). The CBL usually comprises a relatively thin surface layer, where the gradients of temperature and humidity may be significant, as well as a wellmixed layer with constant potential temperature and saturation deficit. Above the capping inversion of the mixed layer is the undisturbed atmosphere, whose properties are determined by synoptic scale processes. In the closed-box model, the CBL is assumed to have an impermeable lid at a fixed height just above the well-mixed layer. At the ground, the areal latent heat flux is governed by the Penman-Monteith equation, identical to (1), with an areal surface resistance $\left\langle r_{5}\right\rangle$ and an areal aerodynamic resistance $\left\langle r_{a}\right\rangle$. It has been shown (Perrier, 1982; McNaughton and Jarvis, 1983) that for steady forcing $\left(<R_{n}-G\right\rangle,\left\langle r_{s}\right\rangle$ and $\left\langle r_{a}\right\rangle$ kept constant), the potential saturation deficit of the mixed layer tends exponentially to an equilibrium value given by

$$
D_{0}=\left(\gamma / \rho c_{p}\right) \frac{s}{s+\gamma}\left\langle R_{n}-G\right\rangle\left\langle r_{s}\right\rangle
$$

and the areal actual evaporation tends to the equilibrium evaporation

$$
\lambda E_{0}=\frac{s}{s+\gamma}\left\langle R_{n}-G\right\rangle
$$

The potential evaporation, as defined by Eqn. (4), will tend to the following equilibrium value (Lhomme, 1997)

$$
E_{p 0}=\alpha_{0} E_{0} \text { with } \alpha_{0}=1+\frac{\gamma}{s+\gamma} \frac{\left\langle r_{s}\right\rangle}{r_{a}}
$$

$r_{a}$ is the aerodynamic resistance of the saturated area, which is assumed to have the same available energy as that of the whole area, and $\alpha_{0}$ is a coefficient similar to the PriestleyTaylor coefficient. This equation (valid only at equilibrium) predicts that the drier a region $(\langle r\rangle$ high), the greater the potential evaporation. It shows clearly the feedback effect (through $<r_{5}>$ ) of areal evaporation on potential evaporation. Equation A3 represents a different formulation of the basic idea contained in the complementary relationship (Bouchet, 1963; Morton, 1983), where potential evaporation is viewed as the effect or the consequence of actual evaporation and as such, as a negative index of actual evaporation.

The air characteristics used as input in the Penman Eqn. (4) are taken in the well-mixed layer (at least between 50 and $100 \mathrm{~m}$ above the ground), which is representative of the regional scale. Then, potential evaporation calculated in this way should be qualified as regional. An approximate physical representation of the saturated surface can be obtained from Eqn. (5): the fetch $x$ needed for the IBL to reach a depth of $50 \mathrm{~m}$ is at least $750 \mathrm{~m}$ in the case of a stand of grass. Therefore, the minimum downwind dimension of the surface maintained at potential rate must be $750 \mathrm{~m}$. For this saturated surface not to alter, substantially, the characteristics of the Convective Boundary Layer in equilibrium with the surface, one may suppose that it must not represent more than $5 \%$ of the whole surface. This means that the minimum downwind dimension of the region influencing the CBL is about $15 \mathrm{~km}$.

\section{References}

Bouchet, R.J., 1963. Evapotranspiration réelle et potentielle, signification climatique. IAHS, Publ. 62, 134-142. 


\section{J.-P. Lhomme}

Brutsaert, W., 1982. Evaporation into the atmosphere. D. Reidel Pub. Co., Dordrecht.

de Bruin, H.A.R., 1989. Physical aspects of the planetary boundary layer with special reference to regional evapotranspiration. In : Estimation of Areal Evapotranspiration edited by T. A. Blade et al. IAHS, Publ. 177.

Doorenbos, J. and Pruitt, W.O., 1977. Crop water requirements. FAO Irrigation and Drainage Paper 24, FAO, Rome.

Garratt, J.R., 1994a. The Atmospheric Boundary Layer. Cambridge University Press. 316 pp.

Garratt, J.R., 1994b. Potential evaporation-Some comments. Internal note, CSIRO.

Granger, R.J., 1989. An examination of the concept of potential evaporation. 7. Hydrol., 111, 9-19.

Jensen, M.E, Burman, R.D. and Allen, R.G., 1990. Evapotranspiration and Irrigation Water Requirements. ASCE Manual 70.

Lhomme, J.P., 1997. A theoretical basis for the Priestley-Taylor coefficient. Boundary Layer Meteorol., 82, 179-191.

McNaughton, K.G. and Jarvis, P.G., 1983. Predicting the effects of vegetation changes on transpiration and evaporation. In T.T. Kozlowski (Editor), Water Deficits and Plant Growth, Vol.VII. Academic Press, London.

Miyake, M., 1965. Transformation of the atmospheric boundary layer over inhomogeneous surfaces, Science report 5R-6, University of Washington, Seattle.

Monteith, J.L., 1965. Evaporation and the environment. Symp. Soc. Exp. Biol., vol. 19, 205-234.

Monteith, J.L., 1981. Evaporation and surface temperature. Quart. 7. Roy. Meteorol. Soc., 107, 1-27.

Morton, F.I., 1983. Operational estimates of areal evapotranspiration and their significance to the science and practice of hydrology. 7. Hydrol., 66, 1-76.
Nash, J.E., 1989. Potential evaporation and 'the complementary relationship'. 7. Hydrol., 111, 1-7.

Penman, H.L., 1948. Natural evaporation from open water, bare soil and grass. Proc. Roy. Soc. London, Ser. A, 193, 120-145.

Penman, H.L., 1956. Evaporation: an introductory survey. Netherlands 7. Agric. Sci., vol 1, 9-29.

Penman, H.L., 1963. Vegetation and Hydrology. Tech. Comm. 53, Commonwealth Bureau of Soils, Harpenden, England.

Perrier, A., 1977. Projet de définitions concernant l'évapotranspiration en fonction de considérations théoriques et pratiques. La Météorologie, VIe série, 11, 7-16.

Perrier, A., 1982. Land surface processes: vegetation. In: P.S. Eagleson (Editor) Land Surface Processes in Atmospheric General Circulation Models. Cambridge University Press.

Priestley; C.H.B. and Taylor, R.J., 1972. On the assessment of surface heat flux and evaporation using large -scale parameters. Mon. Wea. Rev., 100, 81-92.

Shuttleworth, W.J., 1993. Evaporation. In: D.R. Maidment (Editor), Handbook of Hydrology. McGraw-Hill, New York.

Shuttleworth, W.J. and Wallace, J.S., 1985. Evaporation from sparse crops - an energy combination theory. Quart. 7. Roy. Meteorol. Soc., 111, 839-855.

Thom, A.S. and Oliver, H.R., 1977. On Penman's equation for estimating regional evaporation. Quart. 7. Roy. Meteorol. Soc., $103,345-357$.

Thornthwaite, C.W., 1948. An approach toward a rational classification of climate. Geogr. Rev., 38, 55-94.

Wallace, J.S., 1995. Calculating evaporation: resistance to factors. Agric. For. Meteorol., 73, 353-366.

World Meteorological Organization, 1974. International Glossary of Hydrology. WMO Rep. $\mathrm{n}^{\circ} 385,393 \mathrm{pp}$. 